\title{
Efficacy and tolerability of bevacizumab plus capecitabine as first-line therapy in patients with advanced hepatocellular
}

\section{carcinoma}

\section{C-H Hsu ${ }^{1,8}$, T-S Yang ${ }^{2}$, C Hsu ${ }^{1,8}$, HC Toh ${ }^{3}$, RJ Epstein ${ }^{4}$, L-T Hsiao ${ }^{5}$, P-J Chen ${ }^{6}$, Z-Z Lin', T-Y Chao7 and A-L Cheng ${ }^{* 1,6}$}

'Department of Oncology, National Taiwan University Hospital, 7 Chung-Shan South Road, Taipei 10002, Taiwan; ${ }^{2}$ Division of Haematology and Oncology, Departmet of Internal Medicine, Chang Gung Medical Foundation, LinKuo-Branch, 5, Fusing St., Gueishan Township, Taoyuan County 333, Taiwan; ${ }^{3}$ Department of Medical Oncology, National Cancer Center, II Hospital Drive, 1696 10, Singapore, Singapore; ${ }^{4}$ Department of Medicine, University of Hong Kong, Queen Mary Hospital, 102 Pokfulam Road, Hong Kong, China; ${ }^{5}$ Department of Hematology and Oncology, Taipei Veterans General Hospital, No. 201, Sec. 2, Shih-Pai Road, Taipei II 2, Taiwan; ' Department of Internal Medicine, National Taiwan University Hospital, 7 ChungShan South Road, Taipei 10002, Taiwan; 'Division of Hematology and Oncology, Tri-Senvice General Hospital, No. 325, Sec. 2, Chenggong Road, Neihu District, Taipei City I 4, Taiwan

BACKGROUND: Molecularly targeted agents with anti-angiogenic activity, including bevacizumab, have demonstrated clinical activity in patients with advanced/metastatic hepatocellular carcinoma (HCC). This multicentre phase II study involving patients from several Asian countries sought to evaluate the safety and efficacy of bevacizumab plus capecitabine in this population.

METHODS: Histologically proven/clinically diagnosed advanced HCC patients received bevacizumab $7.5 \mathrm{mg} \mathrm{kg}^{-1}$ on day I and capecitabine $800 \mathrm{mg} \mathrm{m}^{-2}$ twice daily on days I - 14 every 3 weeks as first-line therapy.

RESULTS: A total of 45 patients were enrolled; 44 (96\%) had extrahepatic metastasis and/or major vessel invasion and 30 (67\%) had hepatitis B. No grade 3/4 haematological toxicity occurred. Treatment-related grade 3/4 non-haematological toxicities included diarrhoea $(n=2,4 \%)$, nausea/vomiting $(n=1,2 \%)$, gastrointestinal bleeding $(n=4,9 \%)$ and hand-foot syndrome $(n=4,9 \%)$. The overall response rate (RECIST) was $9 \%$ and the disease control rate was $52 \%$. Overall, median progression-free survival (PFS) and overall survival (OS) were 2.7 and 5.9 months, respectively. Median PFS and OS were 3.6 and 8.2 months, respectively, for Cancer of the Liver Italian Programme (CLIP) score $\leqslant 3$ patients, and 1.4 and 3.3 months, respectively, for CLIP score 4 patients. CONCLUSION: The bevacizumab-capecitabine combination shows good tolerability and modest anti-tumour activity in patients with advanced HCC.

British Journal of Cancer (2010) 1 02, 98I-986. doi:10.1038/sj.bjc.6605580 www.bjcancer.com

Published online 16 February 2010

(c) 2010 Cancer Research UK

Keywords: bevacizumab; capecitabine; hepatocellular carcinoma

Primary liver cancer, which consists predominantly of hepatocellular cancer (HCC), is the fifth most common cancer worldwide and the third most common cause of cancer mortality (El-Serag and Rudolph, 2007). More than $80 \%$ of cases occur in East Asia and sub-Saharan Africa, although the incidence is increasing in parts of Europe (Capocaccia et al, 2007) and the United States (El-Serag and Rudolph, 2007). Patients with locally advanced or metastatic HCC face a dismal outcome. Conventional chemotherapy is rarely successful and often poorly tolerated (Palmer et al, 2004; Zhu, 2006a). In the past, the median survival for these

\footnotetext{
*Correspondence: Dr A-L Cheng; E-mail: alcheng@ntu.edu.tw

This study was presented in part at the 44th Annual Meeting of the American Society of Clinical Oncology, Chicago, IL, USA, 30 May-3 June 2008.

${ }^{8}$ These two authors contributed equally to this work.

Received 10 September 2009; revised 22 January 2010; accepted 25 January 2010; published online 16 February 2010
}

patients diagnosed in Asia was typically 2-4 months with best supportive care (Hsu et al, 2010).

Recently, sorafenib, a multikinase inhibitor with anti-angiogenic activity (Wilhelm et al, 2004, 2006), demonstrated a survival benefit $v s$ best supportive care in patients with advanced HCC in two randomised placebo-controlled trials, one conducted in Europe, North America, South America and Australasia (the SHARP study, Llovet et al, 2008b) and the other in the Asia-Pacific region (Cheng et al, 2009). Both trials reported similar objective response rates (2$3 \%)$ and disease control rates (35-43\%); overall survival (OS) was 10.7 months in the SHARP trial (Llovet et al, 2008b) and 6.5 months in the Asia-Pacific trial (Cheng et al, 2009). Although the survival benefit conferred by sorafenib in advanced HCC is an encouraging development, there is still a substantial unmet medical need for effective treatment options with favourable safety profiles in this difficult-to-treat population.

Hepatocellular carcinomas are known to be highly vascularised, with elevated vascular endothelial growth factor (VEGF) and microvessel density levels (Yamaguchi et al, 1998; Pang and Poon, 
2006). The VEGF and VEGFR signalling pathways are the prime targets for developing anti-angiogenic therapy as cancer therapeutics. Bevacizumab is an anti-VEGF antibody that targets tumour angiogenesis and has proven benefit in many solid tumours (Hurwitz et al, 2004; Miller et al, 2005; Sandler et al, 2006); bevacizumab has also been investigated in advanced HCC in a phase II setting. As a single agent, bevacizumab had an objective tumour response rate of $13 \%$ in patients with unresectable nonmetastatic HCC (Siegel et al, 2008). The combination of bevacizumab with standard chemotherapy - a strategy that has demonstrated clinical benefit in patients with colorectal, lung and breast cancer (Hurwitz et al, 2004; Miller et al, 2005; Sandler et al, 2006) - has also been tested in patients with HCC. In phase II studies, the combination of bevacizumab with gemcitabineoxaliplatin (GEMOX) (Zhu et al, 2006b) or capecitabineoxaliplatin (XELOX) (Sun et al, 2007) resulted in response rates of up to $20 \%$, although these regimens were associated with significant treatment-related toxicity. To have a wider application, bevacizumab combinations with a better therapeutic index need to be developed.

Capecitabine, an oral fluoropyrimidine with a favourable safety profile, has been widely used in various types of solid cancers (Roche Products Limited, 2009). Theoretically, capecitabine may have an advantage when treating patients with an abnormal liver function. Indeed, Twelves et al (1999) demonstrated that mild to moderate hepatic dysfunction has no clinically significant influence on the pharmacokinetic parameters of capecitabine and its metabolites. In a retrospective analysis, capecitabine monotherapy resulted in a response rate of $11 \%$ in patients with advanced HCC (Patt et al, 2004).

The combination of bevacizumab with capecitabine, two drugs that have previously demonstrated single-agent activity in patients with advanced HCC, has not yet been evaluated in patients with advanced HCC. The present phase II study was conducted at multiple Asian centres to evaluate the tolerability and efficacy of bevacizumab plus capecitabine in patients with advanced or metastatic HCC.

\section{PATIENTS AND METHODS}

\section{Patients}

Patients aged $\geqslant 18$ years were included if they had histologically confirmed or clinically diagnosed HCC (typical imaging findings plus $\alpha$-fetoprotein $\geqslant 400 \mathrm{ng} \mathrm{ml}^{-1}$ ); stage IV disease (American Joint Committee on Cancer, 1998) that was inoperable and not amendable by other locoregional therapies; Child-Pugh class A; Karnofsky performance status $\geqslant 70 \% ; \geqslant 1$ measurable lesion; neutrophil count $\geqslant 2000$ per $\mu \mathrm{l}$; platelet count $\geqslant 150000$ per $\mu \mathrm{l}$; alanine aminotransferase level $\leqslant 5 \times$ upper normal limit (UNL); or bilirubin $\leqslant 1.2 \times$ UNL. Exclusion criteria included earlier radiotherapy/systemic therapy for advanced HCC; central nervous system metastases; previous HCC rupture; and clinically significant cardiovascular disease. Patients with a history of gastrointestinal bleeding within 1 year or known oesophageal/gastric varices were required to undergo gastroduodenoscopy to exclude active bleeding and a high risk of bleeding.

The study conformed to the principles of the Declaration of Helsinki and Good Clinical Practice Guidelines, with approval obtained from each centre's independent ethics committee. Patients provided written informed consent.

\section{Treatment}

Patients received intravenous bevacizumab $7.5 \mathrm{mg} \mathrm{kg}^{-1}$ on day 1 and oral capecitabine $800 \mathrm{mg} \mathrm{m}^{-2}$ twice daily on days $1-14$ every 3 weeks. The dose of capecitabine was selected on the basis of several observations indicating that capecitabine given in lower doses demonstrated an improved therapeutic index (Sakamoto et al, 2004; Hennessy et al, 2005). For example, in a Japanese cohort of advanced colorectal cancer patients, capecitabine at a dose of $828 \mathrm{mg} \mathrm{m}^{-2}$ twice daily resulted in an improved toxicity profile without compromising its anti-tumour activities (Sakamoto et al, 2004). Six treatment cycles were planned, but patients maintaining a response or stable disease after six cycles could continue treatment at the investigator's discretion.

No dose reduction of bevacizumab was allowed, except for patients with $>10 \%$ change in body weight during the treatment period. Bevacizumab was discontinued in patients with gastrointestinal perforation, arterial thrombotic toxicity, grade $3 / 4$ haemorrhagic toxicity, symptomatic grade 4 thromboembolic toxicity, hypertensive crisis or nephrotic syndrome. Bevacizumab was withheld for grade 3 hypertension and repeated $24 \mathrm{~h}$ proteinuria $>2 \mathrm{~g}$ until these symptoms improved. Capecitabine doses were reduced by $20 \%$ for patients who experienced a first occurrence of a grade 3 haematological toxicity, a second occurrence of grade 2 non-haematological toxicity or any grade 3 non-haematological toxicity. Capecitabine doses were reduced by $40 \%$ for a first occurrence of grade 4 haematological toxicity, a second occurrence of grade 3 haematological toxicity, any grade 4 non-haematological toxicity, a second occurrence of grade 3 non-haematological toxicity or a third occurrence of grade 2 non-haematological toxicity. Capecitabine was discontinued after the occurrence of a second grade 4 haematological toxicity, a third grade 3 haematological toxicity, a fourth grade 2 non-haematological toxicity, a third grade 3 nonhaematological toxicity or a second grade 4 non-haematological toxicity.

\section{Assessments}

Tumour assessment according to the Response Evaluation Criteria in Solid Tumors (RECIST) was performed every 6 weeks using computed tomography/magnetic resonance imaging. Adverse events (AEs) were graded using National Cancer Institute Common Terminology Criteria for Adverse Events, version 3 (National Cancer Institute, 2008).

\section{Statistical analyses}

The null hypothesis (H0) was that the overall response rate (ORR) was $\leqslant 10 \%$, that is, low activity. The alternative hypothesis (H1) was that ORR was $\geqslant 25 \%$, that is, encouraging activity. To distinguish between an ORR of 10 and $25 \%$, assuming $\alpha$ - and $\beta$-error rates of 0.05 and 0.20 , respectively, a sample size of 43 patients was required using Ensign's three-stage design (Ensign et al, 1994). Stage 1 enrolled nine patients and would stop if no responses were confirmed. Stage 2 would enrol 25 patients (including the nine patients from stage 1) and would stop if $\leqslant$ three responses were confirmed. If sufficient responses were observed in stages 1 and 2, the trial proceeded to stage 3 , recruiting 45 patients in total.

The primary objective of this study was to evaluate the ORR for capecitabine plus bevacizumab in patients with advanced/metastatic HCC. ORR was assessed in the per-protocol population, which excluded patients who did not have adequate baseline or $\geqslant 1$ follow-up tumour assessment. Secondary objectives included safety, disease control rate, progression-free survival (PFS) and OS. Secondary analyses were performed on the intent-to-treat population. The PFS and OS were analysed using Kaplan-Meier plots and presented as median event times with $95 \%$ confidence intervals (CIs). 


\section{RESULTS}

\section{Patients}

Between May 2005 and August 2006, 45 Asian patients were enrolled at eight centres in Taiwan, Singapore and Hong Kong. Patients' baseline characteristics are shown in Table 1. All patients except one had extrahepatic metastases $(n=31)$ and/or major hepatic vessel invasion $(n=25)$ : thus 44 patients $(98 \%)$ had Barcelona Clinic Liver Cancer (BCLC) stage C cancer and one had BCLC stage B disease. The most common disease sites were the liver (91\%), lung (47\%) and lymph nodes (31\%).

Patients received a median of three cycles (range 1-31 cycles) of bevacizumab plus capecitabine: 38 patients $(87 \%)$ completed two cycles, $21(47 \%)$ completed four cycles and $15(33 \%)$ completed six cycles. Among these 15 patients, seven continued study treatment for between 10 and 31 cycles. The reasons for discontinuation before six cycles were progressive disease in 25 patients, safety concern in four patients (fulminant hepatitis, gastrointestinal bleeding, hypoalbuminaemia and hyponatraemia, respectively), and withdrawal of consent in one patient. The capecitabine dose

Table I Patient characteristics at baseline $(n=45)$

\begin{tabular}{|c|c|}
\hline Characteristic & Value \\
\hline \multicolumn{2}{|l|}{ Sex, n (\%) } \\
\hline Male & $40(89)$ \\
\hline Female & $5(11)$ \\
\hline Median age, years (range) & $54(23-75)$ \\
\hline \multicolumn{2}{|c|}{ Karnofsky performance status, n (\%) } \\
\hline $90-100$ & $36(80)$ \\
\hline 80 & $9(20)$ \\
\hline \multicolumn{2}{|l|}{ Baseline $\alpha$-fetoprotein, n (\%) } \\
\hline$\geqslant 400 \mathrm{ng} \mathrm{ml}^{-1}$ & $32(7 \mid)$ \\
\hline$<400 \mathrm{ng} \mathrm{ml}^{-1}$ & $13(29)$ \\
\hline \multicolumn{2}{|l|}{ AJCC stage, ${ }^{a}$ n (\%) } \\
\hline $\mathrm{IVa}$ & $16(36)$ \\
\hline $\mathrm{IVb}$ & $29(64)$ \\
\hline \multicolumn{2}{|l|}{ BCLC stage, $n(\%)$} \\
\hline B & I (2) \\
\hline C & $44(98)$ \\
\hline \multicolumn{2}{|l|}{ CLIP score, n (\%) } \\
\hline$\leqslant 2$ & $18(40)$ \\
\hline 3 & $16(36)$ \\
\hline 4 & $11(24)$ \\
\hline \multicolumn{2}{|l|}{ Okuda score, n (\%) } \\
\hline 1 & $21(47)$ \\
\hline$\|$ & $24(53)$ \\
\hline \multicolumn{2}{|c|}{ Site of extrahepatic metastases, n (\%) } \\
\hline Lung & $21(47)$ \\
\hline Lymph nodes & $14(31)$ \\
\hline \multicolumn{2}{|c|}{ Prior curative surgery or locoregional therapy, n (\%) } \\
\hline Yes & II (24) \\
\hline No & $34(76)$ \\
\hline \multicolumn{2}{|c|}{ Virology of underlying liver disease, n (\%) } \\
\hline HBV & $30(67)$ \\
\hline $\mathrm{HCV}$ & $8(18)$ \\
\hline Non-B+non-C & $10(22)$ \\
\hline
\end{tabular}

Abbreviations: $\mathrm{BCLC}=$ Barcelona Clinic Liver Cancer; CLIP = Cancer of the Liver Italian Programme; $\mathrm{HBV}=$ hepatitis $\mathrm{B}$ virus; $\mathrm{HCV}=$ hepatitis $\mathrm{C}$ virus. ${ }^{\mathrm{a}}$ American Joint Committee on Cancer (1998) staging system. was reduced in five patients (elevated bilirubin, $n=1$; hand - foot syndrome, $n=2$; grade 3 diarrhoea, $n=1$; vomiting, $n=1$ ). The bevacizumab dose was delayed in three patients as a result of capecitabine toxicity and adjusted in one patient because of weight loss.

\section{Efficacy}

One patient with no follow-up tumour assessment was excluded from the per-protocol population; 44 patients were therefore evaluable for response. Four patients had a partial response and 19 had stable disease for an ORR of $9 \%$ and a disease control rate of 52\% (95\% CI: 36.7-67.5\%; Table 2).

Serum alpha-fetoprotein (AFP) levels were serially monitored. A total of 37 patients who had elevated baseline AFP were eligible for AFP response. AFP response was $24 \%$ if defined by $20 \%$ decline from baseline (Chan et al, 2009) or $13 \%$ if defined by $50 \%$ decline (Chen et al, 2005; Vora et al, 2009).

After a median follow-up of 19.6 months, the median PFS was 2.7 months (95\% CI: $1.5-4.1$ months) and median OS was 5.9 months (95\% CI: 4.1 -9.7 months) in the intent-to-treat population (Figure 1). The 1-year PFS rate was 20\% (95\% CI: $8-32 \%)$ and 1 -year OS was $27 \%$ (95\% CI: 14-40\%). Correlations between several baseline characteristics and PFS/OS were explored. Only Cancer of the Liver Italian Programme (CLIP) score was significantly correlated with outcome: median PFS was higher in patients with CLIP scores $\leqslant 3$ compared with those with a score of 4 (3.6 months (95\% CI: $1.5-6.0$ months) vs 1.4 months (95\% CI: 1.2-3.3 months)), whereas median OS was 8.2 months (95\% CI: 5.0-11.3 months) vs 3.3 months (95\% CI: 2.5-5.2 months), respectively.

\section{Tolerability}

All 45 patients were evaluable for tolerability. The combination of bevacizumab plus capecitabine was generally well tolerated. Grade 3/4 AEs and laboratory abnormalities are shown in Table 3. In total, 43 patients $(96 \%)$ experienced $\geqslant 1$ AEs; 25 patients $(56 \%)$ had AEs that were possibly related to treatment; and 26 patients $(58 \%)$ had AEs that were probably related to treatment. Treatmentrelated grade $3 / 4$ toxicities were diarrhoea $(n=2,4 \%)$, nausea/ vomiting $(n=1,4 \%)$, gastrointestinal bleeding $(n=4,9 \%$, including three patients with oesophageal variceal bleeding), hand-foot syndrome $(n=4,9 \%)$, lower respiratory tract infection $(n=1,2 \%)$ and proteinuria $(n=1,2 \%)$.

A total of 15 patients had serious AEs; most were gastrointestinal in nature $(n=9)$. Three patients withdrew early from the study as a result of AEs. No deaths were attributed to the study treatment.

\section{DISCUSSION}

Targeted therapy with anti-angiogenic activity has become the mainstay of systemic therapy for advanced HCC (National

Table 2 Efficacy of bevacizumab plus capecitabine in patients with hepatocellular carcinoma $(n=44)$

\begin{tabular}{lc}
\hline Outcome & Value \\
\hline Overall response rate $(95 \% \mathrm{Cl}), \%$ & $9.1(2.5-21.7)$ \\
Complete response, $n(\%)$ & $0(0)$ \\
Partial response, $n(\%)$ & $4(9.1)$ \\
Stable disease, $n(\%)$ & $19(43)$ \\
Progressive disease, $n(\%)$ & $21(48)$ \\
Disease control rate $(95 \% \mathrm{Cl}), \%$ & $52.3(36.7-67.5)$ \\
\hline
\end{tabular}

Abbreviation: $\mathrm{Cl}=$ confidence interval. 
A

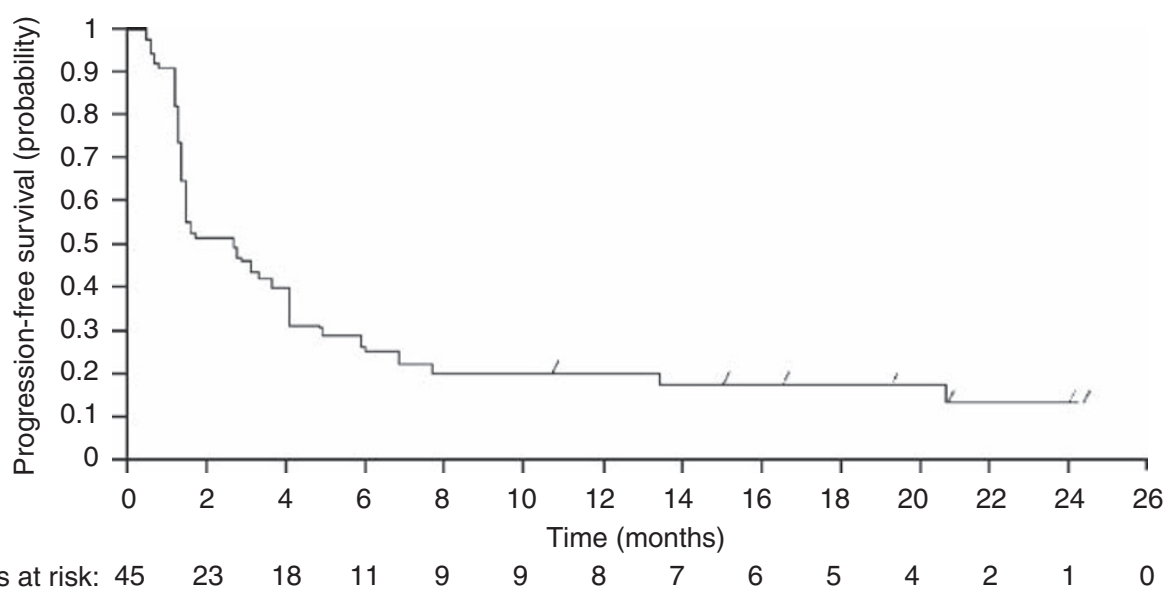

B

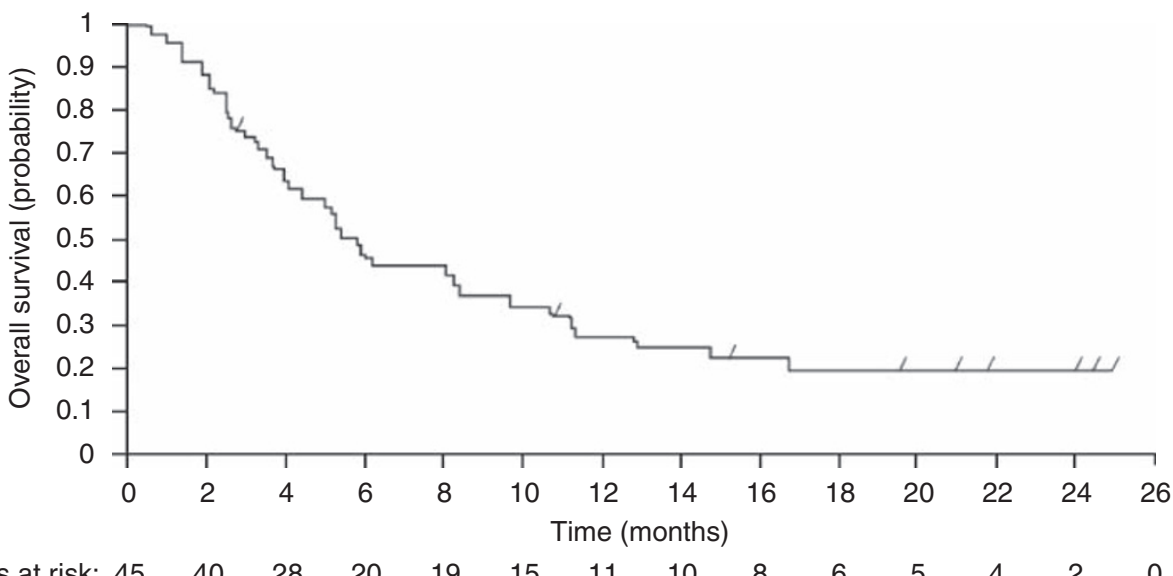

Figure I Duration of progression-free survival $(\mathbf{A})$ and overall survival $(\mathbf{B})$ in patients treated with capecitabine and bevacizumab $(n=45)$.

Table 3 Adverse events in patients with hepatocellular carcinoma treated with capecitabine plus bevacizumab $(n=45)$

\begin{tabular}{lccc}
\hline & \multicolumn{3}{c}{ No. of patients (\%) } \\
\cline { 2 - 4 } Event & All grades & Grade 3 & Grade 4 \\
\hline Adverse events & & & \\
$\quad$ Hand-foot syndrome & $15(33)$ & $4(9)$ & $0(0)$ \\
Nausea & $7(16)$ & $1(2)$ & $0(0)$ \\
Vomiting & $6(13)$ & $1(2)$ & $0(0)$ \\
Diarrhoea & $12(27)$ & $2(4)$ & $0(0)$ \\
Gastrointestinal bleeding & $4(9)$ & $2(4)$ & $2(4)$ \\
Proteinuria & $2(4)$ & $1(2)$ & $0(0)$ \\
Lower respiratory tract infection & $1(2)$ & $1(2)$ & $0(0)$ \\
Fulminant hepatitis & $1(2)$ & $0(0)$ & $1(2)$ \\
Mucositis & $5(11)$ & $0(0)$ & $0(0)$ \\
Skin pigmentation & $3(7)$ & $0(0)$ & $0(0)$ \\
& & & \\
Laboratory values & $12(27)$ & $2(4)$ & $2(4)$ \\
Anaemia & $1(2)$ & $0(0)$ & $0(0)$ \\
Neutropenia & $12(27)$ & $0(0)$ & $0(0)$ \\
Thrombocytopenia & & & \\
Increased hepatic transaminases & $12(27)$ & $0(0)$ & $1(2)$ \\
$\quad$ ALT & $20(44)$ & $6(13)$ & $1(2)$ \\
AST & $30(67)$ & $5(11)$ & $0(0)$ \\
Hyperbilirubinaemia &
\end{tabular}

Abbreviations: $\mathrm{ALT}=$ alanine aminotransferase; $\mathrm{AST}=$ aspartate aminotransferase.
Comprehensive Cancer Network, 2007). Previous studies have shown that bevacizumab is also effective in this difficult-to-treat indication (Zhu et al, 2006b; Sun et al, 2007; Siegel et al, 2008). Further evidence of this activity has been demonstrated in this study. However, the study did not meet the hypothesis that ORR would be $\geqslant 25 \%$, although ORR, combined with PFS and OS, suggests activity for this combination. Retrospectively, it has been recognised that ORR may not be an informative end point in studies of targeted agents for advanced HCC (Llovet et al, 2008a).

At first glance, the efficacy results reported here are lower than those previously reported in bevacizumab-chemotherapy combination trials (Zhu et al, 2006b; Sun et al, 2007). Nevertheless, comparisons between studies of advanced HCC are complicated by differences in recruitment criteria and study designs, as well as by differences in baseline patient characteristics, in particular the proportion of Asian patients in the studies. This is illustrated by two recent sorafenib studies: the SHARP study, which enrolled patients exclusively from Europe and North America (Llovet et al, 2008b), and the Asia-Pacific study, which enrolled patients exclusively from China, Korea and Taiwan (Cheng et al, 2009). Both studies used the same eligibility criteria and were conducted in parallel; although clinical improvement was comparable in the two studies, overall outcomes were much poorer in patients in the Asia-Pacific study. Specifically, median OS was 4.2 and 6.5 months for placebo- and sorafenib-treated patients in the Asia-Pacific study, respectively, compared with 7.9 and 10.7 months in the 
SHARP study. Notably, previously reported clinical studies of bevacizumab with or without chemotherapy in advanced HCC patients were all reported from North America (Zhu et al, 2006b; Sun et al, 2007; Siegel et al, 2008). Taking the advanced HCC patients enrolled in the Asia-Pacific study of sorafenib as a reference group (Cheng et al, 2009), the efficacy outcomes of the current combination are comparable with those of sorafenib.

The efficacy of the bevacizumab plus capecitabine combination is also likely to have been influenced by the fact that this study included a large proportion of poor-prognosis patients. Almost a quarter of patients had a CLIP score of 4 , which is associated with poor prognosis (Farinati et al, 2000; Grieco et al, 2005; Collette et al, 2008). In a study of survival in patients undergoing palliative treatment, those with CLIP scores of $2-3$ had a median OS of 4.57 months, whereas those with CLIP scores of $4-6$ had a median OS of 1.93 months (Collette et al, 2008). This pattern was also apparent in the present analysis of survival: patients with a CLIP score $\leqslant 3$ had a median PFS of 3.6 months and an OS of 8.2 months compared with 1.4 months and 3.3 months, respectively, for patients with a CLIP score of 4 . These data suggest that CLIP score is an important stratification factor for clinical trials in advanced HCC.

The combination of bevacizumab plus capecitabine was well tolerated in this group of patients with advanced HCC. Grade 3/4 haematological toxicities were not observed and the rate of other grade 3/4 toxicities was low. Gastrointestinal bleeding occurred in four patients, three of whom had oesophageal variceal bleeding. Mandatory gastroduodenoscopy was later incorporated into the screening phase for this study. Other bevacizumab HCC studies also adopted measures to exclude patients at high risk of bleeding (Siegel et al, 2008; Thomas et al, 2009). For example, in the study by Siegel et al (2008), the protocol was amended after a variceal bleed that led to the death of one patient in the safety evaluation phase; the amendment required subsequent patients who had varices before or evidence of varices on computed tomography/ magnetic resonance imaging to undergo endoscopy within 4 weeks of study entry (Siegel et al, 2008).

The favourable toxicity profile of our regimen, compared with other combinations used in HCC, is primarily because of the low toxicity of reduced-dose capecitabine. For comparison, the combination of bevacizumab plus GEMOX was associated with grade $3 / 4$ neutropaenia and thrombocytopaenia in 42 and $9 \%$ of patients, respectively (Zhu et al, 2006b). Bevacizumab plus XELOX was associated with grade 3/4 neutropaenia and thrombocytopaenia in 6 and $12 \%$ of patients, respectively, and with grade $3 / 4$ peripheral neuropathy in $12 \%$ of patients (Sun et al, 2007). More stringent inclusion criteria adopted in our study, including higher levels of baseline neutrophil and platelet, may also contribute to the negligible haematological toxicity in our cohort.

The significance of adding capecitabine to bevacizumab in advanced HCC patients could not be evaluated in the current single-arm study. The single-agent response rate of bevacizumab, as reported by Siegel et al (2008), was 13\%, which was even higher than that of our combination. However, the high response rate of bevacizumab shown in that report could be associated with the following facts: the study excluded patients with extrahepatic metastases; it enrolled patients with different aetiological factors; and used higher doses of bevacizumab in two-thirds of their patients. Further studies are warranted to identify the optimal dose of bevacizumab, as well as more effective combinations of bevacizumab in HCC (Thomas et al, 2009).

In summary, the combination of bevacizumab plus capecitabine shows good tolerability and modest anti-tumour activity in patients with advanced or metastatic HCC. Randomised trials are required to determine whether the combination of chemotherapy and bevacizumab is superior to treatment with bevacizumab alone.

\section{ACKNOWLEDGEMENTS}

The authors thank Dr Ying-Chun Shen (Department of Medical Research, National Taiwan University Hospital) for administrative support and data collection/assembly, Dr Kate Jin, Peter Button, Sonya Wang and Dr Guenther Forster (Roche) and Deirdre Carman (Miller Medical Communications) for their contribution to the preparation of the paper.

\section{REFERENCES}

American Joint Committee on Cancer (1998) AJCC Staging Manual 5th edn, Lippincott Williams \& Wilkins: Philadelphia, PA

Capocaccia R, Sant M, Berrino F, Simonetti A, Santi V, Trevisani F, for the EUROCARE Working Group (2007) Hepatocellular carcinoma: trends of incidence and survival in Europe and the United States at the end of the 20th century. Am J Gastroenterol 102: 1661 -1670; quiz 1660, 1671

Chan SL, Mo FK, Johnson PJ, Hui EP, Ma BB, Ho WM, Lam KC, Chan AT, Mok TS, Yeo W (2009) New utility of an old marker: serial alpha-fetoprotein measurement in predicting radiologic response and survival of patients with hepatocellular carcinoma undergoing systemic chemotherapy. J Clin Oncol 27: 446-452

Chen LT, Liu TW, Chao Y, Shiah HS, Chang JY, Juang SH, Chen SC, Chuang TR, Chin YH, Whang-Peng J (2005) Alpha-fetoprotein response predicts survival benefits of thalidomide in advanced hepatocellular carcinoma. Aliment Pharmacol Ther 22: $217-226$

Cheng AL, Kang YK, Chen Z, Tsao CJ, Qin S, Kim JS, Luo R, Feng J, Ye S, Yang TS, Xu J, Sun Y, Liang H, Liu J, Wang J, Tak WY, Pan H, Burock K, Zou J, Voliotis D, Guan Z (2009) Efficacy and safety of sorafenib in patients in the Asia-Pacific region with advanced hepatocellular carcinoma: a phase III randomized, double-blind, placebo-controlled trial. Lancet Oncol 10: $25-34$

Collette S, Bonnetain F, Paoletti X, Doffoel M, Bouché O, Raoul JL, Rougier P, Masskouri F, Bedenne L, Barbare JC (2008) Prognosis of advanced hepatocellular carcinoma: comparison of three staging systems in two French clinical trials. Ann Oncol 19: 1117-1126

El-Serag HB, Rudolph KL (2007) Hepatocellular carcinoma: epidemiology and molecular carcinogenesis. Gastroenterology 132: 2557 - 2576
Ensign LG, Gehan EA, Kamen DS, Thall PF (1994) An optimal three-stage design for phase II clinical trials. Stat Med 13: $1727-1736$

Farinati F, Rinaldi M, Gianni S, Naccarato R (2000) How should patients with hepatocellular carcinoma be staged? Validation of a new prognostic system. Cancer 89: 2266-2273

Grieco A, Pompili M, Caminiti G, Miele L, Covino M, Alfei B, Rapaccini GL, Gasbarrini G (2005) Prognostic factors for survival in patients with early-intermediate hepatocellular carcinoma undergoing non-surgical therapy: comparison of Okuda, CLIP, and BCLC staging systems in a single Italian centre. Gut 54: $411-418$

Hennessy BT, Gauthier AM, Michaud LB, Hortobagyi G, Valero V (2005) Lower dose capecitabine has a more favorable therapeutic index in metastatic breast cancer: retrospective analysis of patients treated at M. D. Anderson Cancer Center and a review of capecitabine toxicity in the literature. Ann Oncol 16: 1289-1296

Hsu C, Shen YC, Cheng CC, Hu FC, Cheng AL (2010) Geographic difference in survival outcome for advanced hepatocellular carcinoma: implications on future clinical trial design. Contemp Clin Trials 31: 55-61

Hurwitz H, Fehrenbacher L, Novotny W, Cartwright T, Hainsworth J, Heim W, Berlin J, Baron A, Griffing S, Holmgren E, Ferrara N, Fyfe G, Rogers B, Ross R, Kabbinavar F (2004) Bevacizumab plus irinotecan, fluorouracil, and leucovorin for metastatic colorectal cancer. N Engl J Med 350: 2335-2342

Llovet JM, Di Bisceglie AM, Bruix J, Kramer BS, Lencioni R, Zhu AX, Sherman M, Schwartz M, Lotze M, Talwalkar J, Gores GJ, for the Panel of Experts in HCC-Design Clinical Trials (2008a) Design and endpoints of 
clinical trials in hepatocellular carcinoma. I Natl Cancer Inst 100: $698-711$

Llovet JM, Ricci S, Mazzaferro V, Hilgard P, Gane E, Blanc JF, de Oliveira AC, Santoro A, Raoul JL, Forner A, Schwartz M, Porta C, Zeuzem S, Bolondi L, Greten TF, Galle PR, Seitz JF, Borbath I, Häussinger D, Giannaris T, Shan M, Moscovici M, Voliotis D, Bruix J, for the SHARP Investigators Study Group (2008b) Sorafenib in advanced hepatocellular carcinoma. N Engl J Med 359: 378 - 390

Miller KD, Chap LI, Holmes FA, Cobleigh MA, Marcom PK, Fehrenbacher L, Dickler M, Overmoyer BA, Reimann JD, Sing AP, Langmuir V, Rugo HS (2005) Randomized phase III trial of capecitabine compared with bevacizumab plus capecitabine in patients with previously treated metastatic breast cancer. J Clin Oncol 23: 792-799

National Cancer Institute (2008) Adult primary liver cancer treatment PDQ summary. Available from: http://www.cancer.gov/cancertopics/pdq/ treatment/adult-primary-liver/healthprofessional/

National Comprehensive Cancer Network (2007) Clinical practice guidelines in oncology. Hepatobillary cancers. Version 1. http://www.nccn.org/ professionals/physician_gls/PDF/hepatobillary.pdf

Palmer DH, Hussain SA, Johnson PJ (2004) Systemic therapies for hepatocellular carcinoma. Expert Opin Invest Drugs 13: 1555-1568

Pang R, Poon RT (2006) Angiogenesis and antiangiogenic therapy in hepatocellular carcinoma. Cancer Lett 242: 151-167

Patt YZ, Hassan MM, Aguayo A, Nooka AK, Lozano RD, Curley SA, Vauthey JN, Ellis LM, Schnirer II, Wolff RA, Charnsangavej C, Brown TD (2004) Oral capecitabine for the treatment of hepatocellular carcinoma, cholangiocarcinoma, and gallbladder carcinoma. Cancer 101: 578-586

Roche Products Limited (2009) Xeloda summary of product characteristics. Available from http://emc.medicines.org.uk/medicine/4619

Sakamoto J, Kondo Y, Takemiya S, Sakamoto N, Nishisho I, Clinical Study Group of Capecitabine (2004) A phase II Japanese study of a modified capecitabine regimen for advanced or metastatic colorectal cancer. Anticancer Drugs 15: 137-143

Sandler A, Gray R, Perry MC, Brahmer J, Schiller JH, Dowlati A, Lilenbaum R, Johnson DH (2006) Paclitaxel-carboplatin alone or with bevacizumab for non-small-cell lung cancer. N Engl J Med 355: 2542 -2550

Siegel AB, Cohen EI, Ocean A, Lehrer D, Goldenberg A, Knox JJ, Chen H, Clark-Garvey S, Weinberg A, Mandeli J, Christos P, Mazumdar M, Popa E, Brown Jr RS, Rafii S, Schwartz JD (2008) II Phase trial evaluating the clinical and biologic effects of bevacizumab in unresectable hepatocellular carcinoma. J Clin Oncol 26: 2992-2998

Sun W, Haller DG, Mykulowycz K, Rosen M, Soulen M, Capparo M, Faust T, Giantonia B, Olthoff K (2007) Combination of capecitabine and oxaliplatin with bevacizumab in treatment of advanced hepatocellular carcinoma: a phase II study. J Clin Oncol 25(18S): (abstr 4574)

Thomas MB, Morris JS, Chadha R, Iwasaki M, Kaur H, Lin E, Kaseb A, Glover K, Davila M, Abbruzzese J (2009) Phase II trial of the combination of bevacizumab and erlotinib in patients who have advanced hepatocellular carcinoma. J Clin Oncol 27: 843-850

Twelves C, Glynne-Jones R, Cassidy J, Schüller J, Goggin T, Roos B, Banken L, Utoh M, Weidekamm E, Reigner B (1999) Effect of hepatic dysfunction due to liver metastases on the pharmacokinetics of capecitabine and its metabolites. Clin Cancer Res 5: 1696-1702

Vora SR, Zheng H, Stadler ZK, Fuchs CS, Zhu AX (2009) Serum alphafetoprotein response as a surrogate for clinical outcome in patients receiving systemic therapy for advanced hepatocellular carcinoma. Oncologist 14: 717-725

Wilhelm S, Carter C, Lynch M, Lowinger T, Dumas J, Smith RA, Schwartz B, Simantov R, Kelley S (2006) Discovery and development of sorafenib: a multikinase inhibitor for treating cancer. Nat Rev Drug Discov 5: $835-844$

Wilhelm SM, Carter C, Tang L, Wilkie D, McNabola A, Rong H, Chen C, Zhang X, Vincent P, McHugh M, Cao Y, Shujath J, Gawlak S, Eveleigh D, Rowley B, Liu L, Adnane L, Lynch M, Auclair D, Taylor I, Gedrich R, Voznesensky A, Riedl B, Post LE, Bollag G, Trail PA (2004) BAY 43-9006 exhibits broad spectrum oral antitumor activity and targets the RAF/ MEK/ERK pathway and receptor tyrosine kinases involved in tumor progression and angiogenesis. Cancer Res 64: 7099-7109

Yamaguchi R, Yano H, Iemura A, Ogasawara S, Haramaki M, Kojiro M (1998) Expression of vascular endothelial growth factor in human hepatocellular carcinoma. Hepatology 28: 68-77

Zhu AX (2006a) Systemic therapy of advanced hepatocellular carcinoma: how hopeful should we be? Oncologist 11: 790-800

Zhu AX, Blaszkowsky LS, Ryan DP, Clark JW, Muzikansky A, Horgan K, Sheehan S, Hale KE, Enzinger PC, Bhargava P, Stuart K (2006b) Phase II study of gemcitabine and oxaliplatin in combination with bevacizumab in patients with advanced hepatocellular carcinoma. J Clin Oncol 24: $1898-1903$ 